\title{
Cutaneous Cryptococcal Infection in Renal Transplant Recipient
}

\author{
Jhansi Vani Devana ${ }^{1}$, Saleem Mohammed Abdul ${ }^{2}$, Vikrantha Reddy ${ }^{3}$ \\ ${ }^{1}$ Department of Microbiology, CARE Hospital, Banjara Hills, Hyderabad, India \\ ${ }^{2}$ Department of General Surgery, CARE Hospital, Banjara Hills, Hyderabad, India \\ ${ }^{3}$ Department of Nephrology, CARE Hospital, Banjara Hills, Hyderabad, India
}

\begin{abstract}
Invasive fungal infections occur in 5-59\% of solid organ transplant recipients. Among fungal infections, Cryptococcus remains a significant opportunistic infection in solid organ transplant recipients. The incidence of cryptococcal infection in solid organ recipients was about 2.8 per 100 transplant recipients. Mortality rates in organ transplant recipients with cryptococcosis range from $15 \%$ to $20 \%$. The type of primary immunosuppressive agents used in solid organ transplant recipients influences the predominant clinical manifestations of cryptococcosis. Patients who are receiving tacrolimus were significantly less likely to have central nervous system involvement and more likely to have skin, soft tissue and osteoarticular involvement than patients receiving non tacrolimus-based immunosuppressive agents We report a case of cutaneous Cryptococcus neoformans infection in 59 years old renal transplant recipient who underwent renal transplantation ten years ago. He was on immunosuppressant drugs like tacrolimus. High clinical suspicion will help in early diagnosis and treatment. J Microbiol Infect Dis 2019; 9(4):164-166.
\end{abstract}

Keywords: Renal transplant recipient, Cryptococcus neoformans, tacrolimus, Amphotericin B

\section{INTRODUCTION}

Invasive fungal infections have been reported in 5 to $59 \%$ of organ transplant recipients. Approximately $8 \%$ of invasive fungal infections in solid organ transplant recipients are due to cryptococcosis and is the third most common invasive fungal infection after candida and Aspergillus. Cryptococcosis has been commonly encountered in HIV-infected patients. There are reports of cryptococcosis in non-HIV-infected patients such as human stem cell transplant recipients, solid organ transplant recipients, patients with haematologic malignancies and patients with other malignancies. Approximately $20-60 \%$ of cryptococcosis in HIV negative patients occurs in solid organ transplant recipients [1]. In an Indian study, $6.1 \%$ of renal transplant recipients were affected by systemic fungal infections. Cryptococcosis was generally a late infectious complication in renal transplant recipients. Cutaneous Cryptococcosis is a manifestation of disseminated infection. CD4+ T- helper responses are critical mediators of immunity against Cryptococcus. Cells of Th1 and Th17 lineage confer protection, T-regulatory cells and Th2 responses compromise host resistance and facilitate disease progression [2]. The cumulative effect of pathogen-induced immunosuppression, withdrawal of immunosuppression and administration of effective antifungal therapy is associated with a shift in the host immune repertoire towards physiologic inflammatory responses that can lead to immune reconstitution syndrome (IRS) [3].

\section{CASE}

A 59-year-old patient admitted to our hospital with complaints of reddish discolouration of right thigh and left calf regions associated with pain and fever of 2 weeks duration. He underwent kidney transplantation 10 years ago. Since then he was on tacrolimus (calcineurin inhibitor) and other Immunosuppressant drugs to prevent

Correspondence: Dr. Jhansi Vani Devana, Department of Microbiology, CARE Hospital, The Institute of Medical Sciences, Banjara Hills

Hyderabad-Telangana, India

Email: devanajhansi@gmail.com

Received: 12 March 2019 Accepted: 11 November 2019

Copyright (C JMID / Journal of Microbiology and Infectious Diseases 2019, All rights reserved 
organ rejection. His vital findings were found stable. There is no history of cough, hemoptysis, dysuria, oliguria, headache or vomiting. There was no lymphadenopathy or hepatosplenomegaly. Local examination of cutaneous lesions revealed $3 \times 3 \mathrm{~cm}$ painful swelling over right thigh and also $2 \times 3 \mathrm{~cm}$ painful swelling over left calf region. Margins are ill defined. Laboratory Investigations showed WBC: 10,500/cumm, random blood sugar: $202 \mathrm{mg} / \mathrm{dl}$, serum creatinine: $3.14 / \mathrm{dl}$, urea: $133 \mathrm{mg} / \mathrm{dl}$, K: 4.8 $\mathrm{mEq} / \mathrm{L}$. 2D Echo and tee transesophageal echo showed $0.5 \mathrm{~cm}^{2}$ mobile mass on aortic valve (suspicious vegetation). Urine and blood cultures did not show any growth. Patient underwent incision and drainage and the pus was sent for microbiological analysis like gram stain, AFB stain, bacterial culture and TB culture. AFB culture did not show any growth of mycobacterium. Gram stain of the pus showed spherical budding yeast cells with halo around the yeast cell [Figure 1].



Figure 1. Gram stain: Gram positive budding yeast cells (100X.

India Ink staining from the sample showed budding yeast cells with a capsule around them. Pus was inoculated on Sabouraud dextrose agar (SDA), Mac Conkey agar and Blood agar. After 48 hrs of incubation, culture showed creamy flat, shiny, moist colonies with smooth edge and was identified as Cryptococcus neoformans [Figure 2]. Cryptococcal antigen titre in the blood was 1:32. Initially he was put on fluconazole, but he did not show any clinical improvement. Tacrolimus was stopped and antifungal agent was upgraded to lipososomal amphotericin B. Haemodialysis was started in view of worsening renal parameters. Gradually he improved with amphotericin B and discharged with same treatment. He was followed up on outpatient basis.

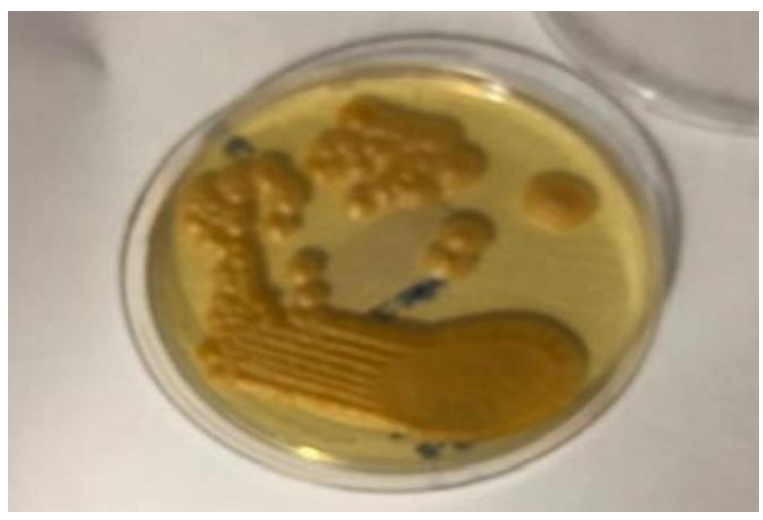

Figure 2. Creamy moist colonies on SDA.

\section{DISCUSSION}

Fungal infections following solid-organ transplantation is a major cause of morbidity and mortality. Cryptococcus neoformans is present in soils enriched by bird excreta, fruits etc. Cutaneous cryptococcal infection may present as erythematous swellings, firm nodules and pustules. The skin is the most common affected site in solid organ transplant recipients who are receiving calcineurin inhibitor like tachrolimus with an incidence of $67 \%$ and $17 \%$ of patients will have meningitis. Calcineurin inhibitors don't appear to influence the incidence but may affect the extent of cryptococcal infection [4]. Cryptococcosis in solid organ transplant recipients is typically a late infectious complication. The median time of onset is 1621 months after transplantation [5]. In our case, the patient came to our hospital with bilateral lower limb cellulitis 10 years after kidney transplantation. Temperature-dependent inhibition of Cryptococcus by tacrolimus may prevent CNS infection but allow the growth of the fungus at cooler body sites such as skin. soft tissue etc. [6]. Our patient who is receiving tacrolimus as an immunosuppressive agent had cutaneous lesions in the lower extremities.

Discontinuation of calcineurin inhibitors was associated with five-fold increased risk of inflammatory reconstitution syndrome (IRS) in transplant recipients with cryptococcal infection. In our case, patient developed renal dysfunction after discontinuation of immunosuppressant drugs and continuation of antifungal therapy. 
Conclusion: Cutaneous cryptococcal infection in solid organ transplant recipients is a late infectious complication. Cutaneous cryptococcal infection frequently mimics and clinically indistinguishable from bacterial cellulitis. There are limited reports on Cutaneous cryptococcal infection in renal transplant recipients in India and to my knowledge there was no study on biologic basis of tissue tropism, reasons for the predominance of dermotrophic strains in recipients who are on tacrolimus in India. Shahid Hussain et al noted a unique propensity of cutaneous cryptococcosis towards extremities and in $94 \%$ of cutaneous C. neoformans infections; the lesions are located on upper or lower extremities. High index of clinical suspicion and early treatment is essential for better outcomes in renal transplant recipients.

\section{ACKNOWLEDGMENTS}

Declaration of Conflicting Interests: The authors declare that they have no conflict of interest.

Funding: This project received no specific grant from any funding agency in the public, commercial, or not-for-profit sectors.

\section{REFERENCES}

1. Vilchez RA, Fung J, Kusne S. Cryptococcosis in organ transplant recipients: an overview. Am J Transplant 2002; 2:575-580

2. Decken K, Kohler G, Palmer-Lehmann K, et al. Interleukin-12 is essential for protective Th1 response in mice infected with Cryptococcus neoformsnans. Infect Immun 1998; 66:4994-5000.

3. Sun HY, Alexander BD, Huprikar S, et al. Predictors of Immune Reconstitution Syndrome in organ transplant recipients with Cryptococcosis: Implications for the management of Immunosuppression. Clin Inf Dis 2015; 60(1):3644.

4. . Singh N, Forrest G. AST Infectious Diseases Community of Practice. Crtptococcosis in solid organ transplant recipients. Am J Transplant 2009; 9 (suppl 4) S192-S198.

5. Pappas PG, Alexander BD, Andes DR, et al. Invasive fungal infections among organ transplant recipients: Results of the Transplant-associated infection surveillance Network (TRANANET) Clin Infect Dis 2010; 50:1101-1011.

6. Agarwal C, Sood V, Kumar A, et al. Cryptococcal infection in transplant kidney manifesting as chronic allograft dysfunction. Indian J Nephrol 2017; 27(5):392-394. 